\title{
AVALIAÇÃo dA ATENÇÃo PRIMÁRIA À SAÚdE NA PERSPECTIVA DOS USUÁRIOS DIANTE DA MUDANÇA DO MODELO ASSISTENCIAL
}

\author{
Marisa Carvalho MARTINS ${ }^{1}$
}

Franciele Fagundes FONSECA ${ }^{2}$

Leonardo de Paula MIRANDA ${ }^{3}$

Patrícia Helena Costa MENDES ${ }^{4}$

\author{
${ }^{1}$ Cirurgiã-dentista. Especialista em Saúde da Família. Universidade Estadual de Montes Claros. \\ marisacv.martins@yahoo.com.br \\ ${ }^{2}$ Enfermeira. Especialista em Saúde da Família. Universidade Estadual de Montes Claros. \\ franciele.fagundes13@gmail.com \\ ${ }^{3}$ Cirurgião-dentista. Mestre em Cuidado Primário em Saúde. Universidade Estadual de Montes Claros. \\ leonardodepaulamiranda@bol.com.br \\ ${ }^{4}$ Cirurgiã-dentista. Doutora em Ciências da Saúde. Universidade Estadual de Montes Claros \\ patyhcmendes@yahoo.com.br
}

Recebido em: 07/05/2016 - Aprovado em: 23/11/2016 - Disponibilizado em: 18/12/2016

\begin{abstract}
RESUMO:
No Brasil, a estratégia adotada pelo Ministério da Saúde para a expansão da Atenção Primária à Saúde (APS) é a Estratégia Saúde da Família (ESF), que por sua vez vem gradualmente substituindo as Unidades Básicas de Saúde Tradicionais (UBS). Neste processo de transição, observam-se alguns avanços, mas também desafios. Este trabalho objetivou avaliar, na perspectiva da satisfação dos usuários, o serviço de APS prestado por equipes da ESF recentemente implantadas em um território tradicionalmente assistido por uma UBS em Montes Claros, Minas Gerais, Brasil. Trata-se de uma investigação de abordagem qualitativa, cujo referencial teórico adotado foi o materialismo histórico-dialético e como referencial metodológico a análise de conteúdo temática. A coleta de dados envolveu a aplicação de entrevistas semiestruturadas a 20 usuários que utilizaram as distintas modalidades de atenção à saúde. A partir da análise das entrevistas emergiram três categorias conceituais e oito subcategorias relacionadas à avaliação dos serviços de atenção primária. Observou-se, de maneira geral, que os usuários estão satisfeitos com o novo modelo, uma vez que apontaram maiores benefícios em relação à acessibilidade, ao acolhimento e à resolubilidade da assistência, quando comparados ao serviço anterior. Avaliar o cuidado à saúde, na perspectiva dos clientes, corrobora com o princípio da participação popular, além de ofertar informações essenciais para a gestão dos serviços prestados.
\end{abstract}

Palavras-chave: Atenção Primária à Saúde. Avaliação de Serviços de Saúde. Estratégia Saúde da Família.

\section{ABSTRACT:}

In Brazil, the strategy adopted by the Ministry of Health for the expansion of Primary Health Care (PHC) is the Family Health Strategy (FHS), which in turn is gradually replacing the traditional Basic Health Units (BHU). In this transition process are observed some progress, but also challenges. This study evaluated in view of user satisfaction, the PHC service provided by the FHS teams recently deployed in a territory traditionally assisted by a BHU in Montes Claros, Minas Gerais, Brazil. This is a qualitative research approach whose theoretical reference was the historical and dialectical materialism and the methodological framework to thematic content analysis. Data collection involved the application of semi-structured interviews with 20 users who used the different modalities of health care. From the analysis of the interviews revealed three conceptual categories and eight subcategories related to the evaluation of primary care services. It was observered, in general, that the users are satisfied with the new model, as showed the greater benefits in relation to accessibility, the reception and the resoluteness of assistance compared to the previous service. Evaluate services from the customer perspective corroborates the principle of popular participation, in addition to offering essential information for management.

Keywords: Primary Health Care. Health Services Evaluation. Family Health Strategy. 


\section{INTRODUÇÃO}

A Atenção Primária à Saúde (APS) no Brasil possui uma história e sua implementação seguiu ciclos em que a incorporação de saberes e o contexto políticoinstitucional definiram prioridades e deram ênfases distintas a seus princípios constituintes (BRASIL, 2013).

A Estratégia Saúde da Família (ESF) emergiu em 1994 e desde o final da década de 1990 vem sendo assumida pelo Ministério da Saúde como a principal estratégia de organização da APS no país (BRASIL, 1998). Desde então, os municípios tem se esforçado para substituir as Unidades Básicas de Saúde Tradicionais (UBS), ou Centros de Saúde, unidades também promotora de cuidados primários, em equipes de ESF, uma transição caracterizada por avanços, mas também por muitas dificuldades e desafios.

As UBS caracterizam-se por unidades formadas por uma equipe multiprofissional, constituídas por médicos clínicos gerais e internistas, pediatras, ginecologistas, enfermeiros e odontólogos. O processo de trabalho é mais voltado para o atendimento da demanda espontânea e em ações curativistas. Já a ESF, possui a noção de adscrição da clientela, é formada por uma equipe multiprofissional contando com médico, enfermeiro, técnico de enfermagem, agente comunitário de saúde podendo haver também profissionais de saúde bucal. (CASTRO et al, 2012). Além disso, a ESF incorpora atividades de promoção, proteção e recuperação da saúde (BRASIL, 1998).

Montes Claros, município localizado no norte do estado de Minas Gerais, desde 1998 iniciou processo de transição do modelo assistencial da APS de forma gradual, substituindo suas 15 UBS, em equipes de saúde da família. Atualmente, o município possui cobertura total de Saúde da Família com 136 equipes.

Pesquisar a respeito da satisfação dos clientes, que experimentam esta transição, é uma tarefa fundamental para a gestão do serviço realizado, uma vez que contribui para a avaliação de desempenho, indicando decisões tanto estratégicas quanto operacionais capazes de influenciar o nível de qualidade dos serviços prestados (MILAN, 2005).

A satisfação com os serviços não é, em si, uma medida de qualidade da atenção. Entretanto, pode estar indiretamente relacionada com a qualidade, porque pode promover a busca por determinados tipos de serviços que modificam o estado de saúde (STARFIELD, 2002).

Nesse contexto, o objetivo do presente estudo foi avaliar, na perspectiva da satisfação dos usuários, os serviços de saúde prestados por equipes da ESF recentemente implantadas em uma área tradicionalmente assistida por uma UBS no município de Montes Claros, Minas Gerais, Brasil. 


\section{METODOLOGIA}

Trata-se de um estudo de delineamento qualitativo, utilizando-se como referencial teórico o materialismo históricodialético e como referencial metodológico a análise de conteúdo temática. O estudo foi realizado entre os meses de novembro de 2015 a fevereiro de 2016 em um serviço de APS prestado por equipes de Saúde da Família recentemente implantadas em território tradicionalmente assistido pela UBS Major Prates em Montes Claros, Minas Gerais, Brasil. Nesta unidade básica de saúde, funcionam quatro equipes da Estratégia Saúde da Família (ESF): ESF Major Prates I, II, III e IV.

A coleta de dados envolveu a aplicação de entrevistas semiestruturadas a 20 usuários cadastrados nas referidas equipes, que possuíam tempo de moradia superior a 5 anos e que utilizaram o serviço público de saúde na modalidade UBS e posteriormente como ESF. O roteiro da entrevista foi elaborado pelos próprios autores, tendo sido testado e adequado a partir da realização de um pré-teste.

As entrevistas aconteceram no domicílio dos usuários e foram aplicadas por dois entrevistadores. As mesmas foram gravadas e transcritas logo após sua realização, a fim de permitir uma melhor análise dos discursos.

Elegeu-se o materialismo históricodialético como referencial teórico para as análises das entrevistas, uma vez que constitui uma importante possibilidade de interpretação do trabalho em saúde.

As informações obtidas com as entrevistas foram submetidas à análise de conteúdo temática, procurando identificar as unidades de significação que se mostrassem relevantes à compreensão da percepção dos usuários em relação à qualidade dos serviços prestados pela unidade de saúde diante da mudança do modelo assistencial.

Desta forma, as etapas da análise de conteúdo temática definidas por Minayo (2013) foram obedecidas. Inicialmente, procedeu-se à etapa de pré-análise, que compreende leituras sucessivas das transcrições para identificação de similaridades e divergências entre as falas para categorização. Realizou-se a exploração do material, com criação de títulos para as categorias e subcategorias emergentes; e, posteriormente, o tratamento dos resultados, que permite confrontar os achados com a literatura científica.

Este estudo foi submetido ao Comitê de Ética em Pesquisa da Universidade Estadual de Montes Claros - MG recebendo parecer consubstanciado de aprovação $\mathrm{n}^{\circ}$ 98607/2015. Todos os indivíduos que participaram da pesquisa assinaram o Termo de Consentimento Livre e Esclarecido para permissão da análise e publicação dos dados. Para garantir o sigilo, os indivíduos são representados pela letra $\mathbf{E}$ (entrevistados) e a 
numeração arábica determina um código estabelecido pelos pesquisadores.

\section{RESULTADOS E DISCUSSÃO}

\section{Caracterização dos participantes}

Dos usuários participantes deste estudo, 2 (10\%) eram homens e 18 (90\%) mulheres. As idades variaram entre 28 a 88 anos e houve uma variação entre 6 a 35 anos em relação ao tempo de moradia no bairro. Após análise de conteúdo temática, surgiram três categorias conceituais e oito subcategorias, conforme mostra a Figura 1.

Figura 1 - Categorias conceituais e subcategorias sobre a avaliação da atenção primária à saúde na perspectiva dos usuários diante da mudança do modelo assistencial em Montes Claros, Minas Gerais, Brasil.

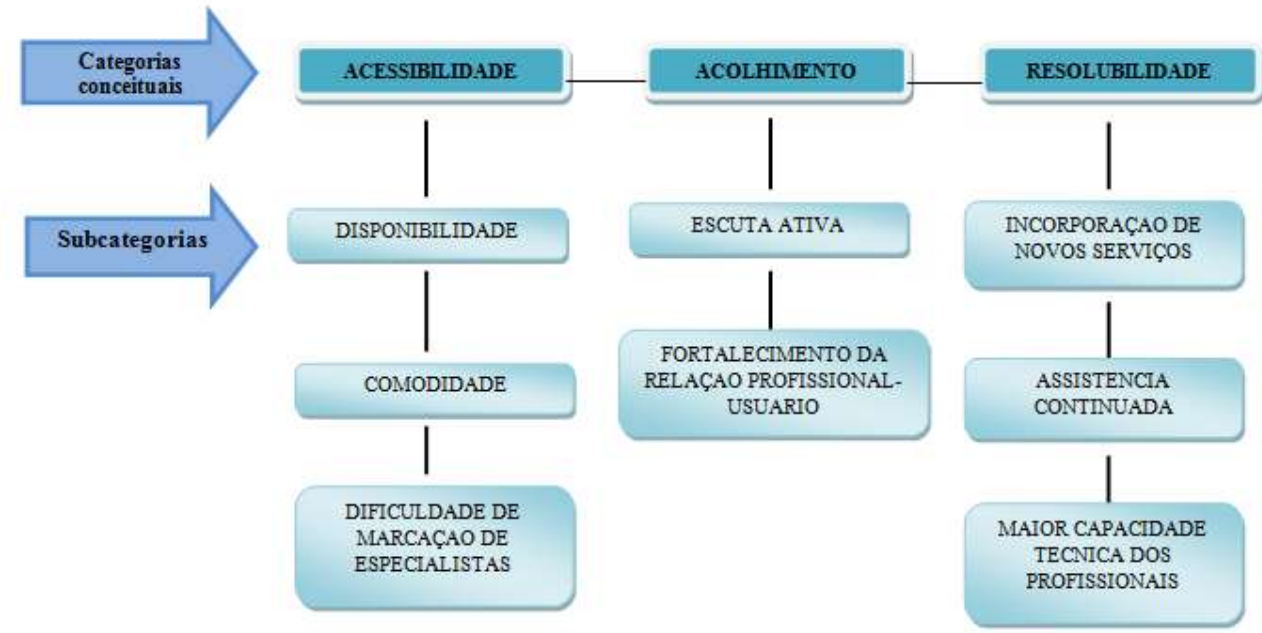

Fonte: Elaborado pelos autores.

\section{Apresentação das categorias e subcategorias}

\section{Categoria 1 - acessibilidade e subcategoria}

\section{1 - disponibilidade}

Para Starfield (2002), a APS deve ser o ponto de entrada de fácil acesso ao usuário para o sistema de serviços de saúde. A autora ressalta que a acessibilidade é indispensável para iniciar o primeiro contato.
Um dos requisitos da acessibilidade é a disponibilidade em ofertar serviços necessários para atender as demandas da população. Penna (2009) afirma que a oferta de serviços deve ser aceitável e adequada às necessidades da população.

Neste estudo, os usuários relataram perceber maior disponibilidade de ofertas de vagas para atendimento médico, como também facilidade de acesso ao cirurgião- 
dentista, profissional que na UBS era mais difícil de obter consulta.

E19: "Antes era só consulta com o médico mesmo e mais nada. Marcar consulta no dentista eu nunca consegui porque sempre que ia lá mandavam voltar no mês seguinte porque já não tinha vaga, Agora consegui."

E4: "Hoje é mais fácil consultar, tem mais vagas e mais pessoas para trabalhar."

\section{Subcategoria 1.2 - comodidade}

A comodidade foi outro fator identificado em relação à acessibilidade. Ela está relacionada ao tempo de espera para o atendimento, a conveniência de horários, a forma de agendamento, a facilidade de contato com os profissionais, o conforto dos ambientes para atendimento, entre outros (MENDES, 2011).

Os usuários destacaram a modificação do sistema de marcação de consultas, outrora por ordem de chegada à unidade e modificada pela triagem, utilizando-se critérios de classificação de risco.

E4: "Nós tivemos mais dificuldades quando era centro de saúde que tinha ficha, acordava de madrugada pra marcar consulta e não conseguia nada."

E5: "Agora, sempre marcam meu pré-natal num horário que eu posso."

E1: "Hoje tem estrutura, a gente vê isso, porque antes aquele posto de saúde estava aos pedaços, agora reformou, está melhor."
Figueiredo et al. (2009) afirmam que o acesso está ligado ao mesmo tempo pela competência de recursos humanos acordados com a estrutura física, definindo quais indivíduos receberão os serviços e em que tempo e condições.

\section{Subcategoria 1.3 - dificuldade de marcação de especialista}

Uma questão abordada pelos usuários nas entrevistas foi a queixa quanto à dificuldade no agendamento de consultas e exames especializados e, ainda, declararam que isso ficou pior com a implantação da ESF.

E4: "Tenho dificuldade de marcar especialistas, demora sair vaga, coloca o nome do caderno e demora muito chamar."

E7: “Às vezes quero consultar com um especialista e não posso, dizem que tenho que consultar com o médico daqui."

Um aspecto importante que deve ser resssaltado é o fato de, apesar de ter sido ampliada a cobertura da atenção básica, ainda haver sérias limitações para o acesso a exames laboratoriais e radiológicos ou para apoio em outras áreas indispensáveis para a continuidade da atenção (SILVA JÚNIOR; ALVES, 2007).

Por outro lado, durante a análise das entrevistas, foi possível identificar que houve uma redução dos encaminhamentos à atenção secundária, fato relacionado intrinsecamente 
com uma maior capacidade de resolução dos problemas por parte dos profissionais da APS. No entanto, isso ainda não é claramente compreendido pelo usuário que ainda deseja consultar prioritariamente com especialistas.

E7: "A única coisa que gostava mais no centro de saúde era a facilidade de marcar a consulta com o especialista, é muito bom ter um especialista perto da gente, tem coisas que o médico generalista não resolve e não está muito bem preparado, o especialista já vai no problema e resolve."

Mendes (2011), afirma que atenção primária apresenta impacto positivo sobre vários indicadores de saúde: reduz gastos; melhora o acesso aos serviços, promove ações preventivas; facilita a detecção precoce de problemas de saúde pública, redução de internações hospitalares; garantia do cuidado longitudinal e reduz a demanda de uso da atenção especializada.

\section{Categoria 2 - acolhimento}

O acolhimento é uma prática presente nas relações de cuidado, nos encontros reais entre trabalhadores de saúde e usuários, nos atos de receber e escutar as pessoas, podendo acontecer de formas variadas (BRASIL, 2013).

Nesta categoria, os usuários
demonstraram perceber uma atitude mais
acolhedora nesta nova modalidade de

assistência ao destacarem, como estratégias diferenciais no cuidado, a escuta ativa por parte dos profissionais, que consequentemente favorece o fortalecimento da relação deste com o usuário.

\section{Subcategoria 2.1 - escuta ativa}

$\mathrm{O}$ acolhimento não se limita apenas a uma recepção cordial, mas extrapola esse conceito, incluindo a escuta ativa do usuário (MOIMAZ, 2010). A escuta é essencial, quando se fala de troca de saberes, em compreensão de mundo e de linguagem, para que se possa entender e ser entendido (MANO, 2004).

E10: "Eu percebi que o saúde da familia agora sabe me receber melhor, parecem estar mais animados, empenhados, isso é bom, deixa a gente melhor. Antes mal as pessoas olhavam no meu rosto, respondiam sem o interesse de ajudar."

Para acolher as demandas dos indivíduos com equidade e qualidade, não necessariamente deve-se encaminhar todas as pessoas ao médico (o acolhimento não deve se restringir a uma triagem para atendimento médico) (BRASIL, 2013). Assim, todos os profissionais da equipe multiprofissional devem exercer a habilidade de ouvir as queixas e se empenhar na resolução das necessidades do usuário. 
Subcategoria 2.2 - fortalecimento da relação profissional-usuário

Garuzi (2014) revelou que o acolhimento constitui um momento de aproximação com o usuário, possibilitando o resgate de valores de solidariedade, cidadania, respeito com o outro e contribui para o estabelecimento de vínculo entre os envolvidos.

E19: "Acho os profissionais mais atenciosos, minha médica até me conhece depois das consultas, lembra de mim, pergunta como estou. Antes era diferente. Não tinha toda essa preocupação."

O atendimento humanizado do paciente proporciona segurança a este e fortifica a relação profissional-paciente, contribuindo inclusive na melhora da saúde do paciente (BRASIL, 2013).

E15: "Gostei do atendimento da médica. Foi muito atenciosa. E eles ficam conhecendo a gente. Dizem que é a médica da família né? Isso é bom, essa aproximação."

E16: "Eu converso facilmente com o médico e ele sabe minha situação e sempre que preciso me ajuda com receitas, remédios e encaminhamentos."

Vale destacar que o estreitamento da relação contribui para desenvolver a qualidade da assistência, visto que, entre outros fatores, isso influencia na aderência do indivíduo ao tratamento da doença crônica, gerando confiabilidade no seguimento de condutas e orientações (CARREIRA; RODRIGUES, 2010).

\section{Categoria 3 - resolubilidade}

Os usuários atribuíram à ESF uma maior capacidade de resolubilidade de suas necessidades quando comparado ao serviço prestado pela antiga UBS. A resolubilidade neste estudo foi associada à implantação de novos serviços, à assistência continuada, além da percepção de uma maior capacidade técnica dos profissionais em relação ao manejo clínico.

\section{Subcategoria 3.1 - incorporação de novos serviços}

Este estudo demonstrou que os usuários estão satisfeitos com a proposta da ESF especialmente pela incorporação de novos serviços, com destaque para a visita domiciliar realizada pela equipe multiprofissional, considerada como uma novidade que contribui para a melhoria da qualidade da assistência prestada.

E8: "Fiz uma cirurgia na época do centro de saúde, mas tinha que ir todo dia no posto fazer curativo. Agora, estou com outro curativo no pé e a enfermeira vem na minha casa fazer o curativo."

E9: "Meu pai é acamado, antes tínhamos muitas dificuldades para levar ele no posto, 
tinha que arrumar um motorista, cadeira de rodas e ele não gostava, agora recebemos o médico em casa."

A incorporação do agente comunitário de saúde também foi apontada como estratégia que facilita a assistência e, portanto, melhora os resultados em saúde:

E3: "Hoje sempre que vou ao posto de saúde, sou atendida de alguma forma. Além disso, hoje tem a agente de saúde que vai na minha casa e pergunta se preciso de algo."

Ademais, outros serviços apontados que contribuem para a maior resolubilidade da ESF foi a inclusão de ações de promoção da saúde e prevenção de doenças, além de consultas realizadas pelo profissional enfermeiro como a puericultura.

E19: "No PSF tem várias consultas que podemos fazer. Agora tem consulta de criança feita pelo enfermeiro, quando nasce uma criança o enfermeiro vai na casa visitar e depois acompanha ela."

E14: "Hoje tem mais cuidado, mais atenção, sem contar que vocês dão palestra, explicam agora, porque antigamente, que palestra? Não existia nada disso, nenhum cuidado, nem orientação e prevenção, nada, agora tem."

\section{Subcategoria 3.2 - assistência continuada}

A assistência continuada é uma das características que compõem a longitudinalidade, que lida com o crescimento e as mudanças de indivíduos no decorrer de um período de anos e está associada a diversos benefícios como a uma menor utilização dos serviços; melhor atenção preventiva; atenção mais oportuna e adequada; menos doenças preveníveis; melhor reconhecimento dos problemas dos usuários; menos hospitalizações e custos totais mais baixos (STARFIELD, 2002).

E2: “Sempre mudava o médico quando era centro de saúde, então eles não conheciam o meu caso e sempre mudavam meus remédios e eu passava mal."

E5: "O cuidado agora está maior, minha glicose ficava sempre alta, mas agora como estou sendo acompanhado, estou conseguindo manter ela controlada."

\section{Subcategoria 3.3 - maior capacidade técnica dos profissionais}

O profissional que atua na Estratégia de Saúde da Família deve ter uma visão holística do usuário e ser capaz de atuar com senso crítico, mediante uma prática humanizada, competente e resolutiva (DAB, 2000).

O melhor desempenho clínico dos profissionais deve-se também à incorporação de tecnologias leves de cuidado como a habilidade de comunicação verbal e não verbal (CERON, 2011), além de um manejo clínico adequado dos problemas de saúde (BRASIL, 1998). 
A ideia da produção de novas tecnologias implica em um empreendimento alicerçado sobre a necessidade, vista como um problema a ser resolvido; sobre o conhecimento, que é o saber que orienta uma nova alternativa para resolver esse problema e, ainda, sobre a criatividade, que é a capacidade de encontrar alternativas para resolver um problema existente (MENDES, 2002).

Ressalta-se também a incorporação da abordagem familiar e comunitária que favorecem a visão sistêmica do indivíduo, de forma que a maior parte dos problemas de saúde pode ser resolvida no primeiro nível de atenção (ALMEIDA et al., 2011).

E1: "Percebi que o médico do PSF soube resolver o meu problema sem precisar me encaminhar para outro”.

E4: "Antes o médico escutava muito pouco o que falava, agora eu consigo falar mais sobre meus problemas."

Após a descrição das categorias e subcategorias, cabe analisar o processo de transição do modelo assistencial sob a perspectiva do materialismo históricodialético. $\mathrm{O}$ materialismo histórico remete ao processo histórico de construção do Sistema Único de Saúde, em que pode-se observar o movimento da luta de classes e as sucessivas tentativas históricas da população que exigiam seus direitos por uma assistência à saúde mais justa e equânime. Além disso, ressalta-se a mudança na organização do processo de trabalho ao longo do tempo, a fim de adequar o sistema de saúde às modificações na concepção do processo saúde-doença. Quanto ao materialismo dialético, Benite (2009) conceitua dialética como um movimento interno de produção da realidade, em que a contradição é vista como foco principal. $\mathrm{Na}$ concepção marxista, a contradição se estabelece entre homens reais em condições históricas e sociais observadas em momentos de mudanças. Neste trabalho, foi possível perceber que ora os usuários se sentem confortáveis com o novo sistema, ora insatisfeitos, o que varia de acordo com o atendimento de suas exigências. Compreender as contradições presentes na percepção dos usuários é de extrema relevância para o planejamento dos serviços à medida que mostra lacunas que precisam ser revistas e trabalhadas com a população.

\section{CONSIDERAÇÕES FINAIS}

A partir do estudo realizado, foi possível perceber que os usuários entrevistados estão mais satisfeitos em relação à prestação dos serviços pelas ESF quando comparado à assistência anterior ofertada pela UBS. A satisfação do usuário pode ser percebida ao apontarem fatores como maior acessibilidade ao serviço, melhor acolhimento, além da percepção de que o 
serviço tornou-se mais resolutivo. Percebe-se que a insatisfação do usuário está relacionada à necessidade de atendimento de especialistas, o que também pode ser atribuído ao fato de a Estratégia Saúde da Família possuir maior resolubilidade, diminuindo assim a demanda pela atenção secundária.

\section{REFERÊNCIAS}

ALMEIDA, Patty Fidelis de. FAUSTO, Márcia Cristina Rodrigues. GIOVANELLA, Lígia. Fortalecimento da atenção primária à saúde: estratégia para potencializar a coordenação dos cuidados. Rev. Panam. Salud Publica. 2011; 29(2):84-95.

BENITE, Anna Maria Canavarro. Considerações sobre o enfoque epistemológico do materialismo históricodialético na pesquisa educacional. Revista Iberoamericana de Educación. Vol. 50, Nº. 4, 2009.

\section{BRASIL. Acolhimento à demanda} espontânea. Brasília: Ministério da Saúde, Cadernos de Atenção Básica, n. 28, 56p. 2013.

BRASIL. Saúde da família: uma estratégia para a reorientação do modelo assistencial. Brasília: Ministério da Saúde; 1998.

CARREIRA, Lígia. RODRIGUES, Rosalina Aparecida Partezani. Dificuldades dos familiares de idosos portadores de doenças crônicas no acesso à Unidade Básica de Saúde. Revista Brasileira de Enfermagem, v. 63, n. 6, p. 933-939, nov.-dez. 2010.

CASTRO, Rodrigo Caprio Leite de. et al. Avaliação da qualidade da atenção primária pelos profissionais de saúde: comparação entre diferentes tipos de serviços. Cad. Saúde Pública, Rio de Janeiro, 28(9):1772-1784, set, 2012.
CERON, Mariane. Habilidades de Comunicação: Abordagem centrada na pessoa. Módulo Psicossocial. UNA-SUS | UNIFESP, 2011. Acesso em: Fev. 2016. Disponível em:

(http://www.unasus.unifesp.br/biblioteca_virt ual/esf/1/.../Unidade_17.)

\section{DEPARTAMENTO DE ATENÇÃO}

BÁSICA (DAB). Informes Técnicos

Institucionais: Programa Saúde da Família. Rev. Saúde Pública 2000; 34(3):316-19.

FIGUEIREDO, Tânia Maria Ribeiro Monteiro de. et al. Desempenho da atenção básica no controle da tuberculose. Revista de Saúde Pública, v. 43, n. 5, p. 825-831, 2009.

GARUZI, Miriane. et al. Acolhimento na Estratégia Saúde da Família: revisão integrativa. Rev. Panam. Salud Pública 35(2), 2014.

MANO, Maria Amélia. A educação em saúde e o PSF resgate histórico, esperança eterna. Boletim da Saúde. Porto Alegre, v.18, n. 1, jan/jul 2004.

MENDES, Eugênio Vilaça. As redes de atenção à saúde. Brasília: OPAS, 2011.

MENDES, Isabel Amélia Costa. et al. A produção tecnológica e a interface com a Enfermagem. Rev. Bras. Enferm. Set-Out 2002; 55 (5): 556-60.

MILAN, Gabriel Sperandio; TREZ, Guilherme. Pesquisa de satisfação: um modelo para planos de saúde. RAE-letrônica, v. 4, n. 2, Art. 17, jul./dez. 2005.

MINAYO, Maria Cecília de Souza. O desafio do conhecimento: pesquisa qualitativa em saúde. 13. ed., São Paulo: Hucitec, 2013.

MOIMAZ, Suzely Adas Saliba. et al. Satisfação e percepção do usuário do SUS sobre o serviço público de saúde. Physis Revista de Saúde Coletiva, Rio de Janeiro, 20 [ 4 ]: 1419-1440, 2010. 
PENNA, Cláudia Maria Mattos. Acesso X

Resolutividade: a perspectiva de quem busca

e de quem oferta atenção à saúde. UFMG,

2009. 18p.

SILVA JÚNIOR, Aluisio Gomes. ALVES,

Carla Almeida. Modelos Assistenciais em

Saúde: desafios e perspectivas. Rio de

Janeiro: EPSJV/Fiocruz, 2007. p 27-41.

STARFIELD, Bárbara. Acessibilidade e

primeiro contato: a porta. Brasília:

UNESCO, Ministério da saúde, 2002.

Pág:207-246. 